\title{
Construction and coupling of frames in Hilbert spaces with $W$-metrics
}

\author{
German Escobar $^{a}$, Kevin Esmeral $^{b}$, Osmin Ferrer $^{b *}$ \\ ${ }^{a}$ Universidad Surcolombiana, Programa de Matemática Aplicada, Neiva, Colombia \\ ${ }^{b}$ Universidad de Sucre, Departamento de Matemáticas, Sincelejo, Colombia.
}

\section{Dedicated to the memory of our dear friend Ricardo Cedeño}

\begin{abstract}
A definition of frames unitarily equivalent in Hilbert spaces with $\mathrm{W}$-metric is stated, and a characterization is given in terms of their respective analysis operators. From a Hilbert space with a frame we construct a Hilbert space with $\mathrm{W}$-metric and a frame unitarily equivalent to the given one. Finally, we prove that the coupling of two frames is a frame.
\end{abstract}

Keywords: Krein spaces, W-spaces, W-metrics, construction of frames, coupling of frames, similar frames.

MSC2010: 42C15, 47B50, 46C20.

\section{Construcción y acoplamiento de marcos en espacios de Hilbert con W-métricas}

Resumen. Se definen marcos unitariamente equivalentes en espacios de Hilbert con W-métricas, y se da una caracterización de ellos comparando sus respectivos operadores de análisis. A partir de un espacio de Hilbert con un marco se construye un espacio de Hilbert con W-métrica y un marco unitariamente equivalente al dado. Finalmente, se muestra que el acoplamiento de dos marcos es un marco.

Palabras clave: Espacios de Krein, W-espacios, W-métricas, construcción de marcos, acoplamiento de marcos, marcos similares.

\footnotetext{
*E-mail: osmin.ferrer@unisucre.edu.co

Received: 18 August 2015, Accepted: 08 March 2016.

To cite this article: G. Escobar, K. Esmeral, O. Ferrer, Construction and coupling of frames in Hilbert spaces with W-metrics, Rev. Integr. Temas Mat. 34 (2016), No. 1, 81-93.
} 


\section{Introduction}

Let $\mathcal{H}$ be a separable Hilbert space. A countable family of vectors $\left\{x_{n}\right\}_{n \in I}$ in $\mathcal{H}$ is a frame if there are constants $0<A \leq B<\infty$ such that for every $x \in \mathcal{H}$

$$
A\|x\|^{2} \leq \sum_{n \in I}\left|\left\langle x, x_{n}\right\rangle\right|^{2} \leq B\|x\|^{2} .
$$

In frame theory the most important result is the Decomposition Theorem [8], [9], [10], [11], [12], [18], [13], [15], [21]. It states that if $\left\{x_{n}\right\}_{n \in I}$ is a frame for $\mathcal{H}$, then every vector $x \in \mathcal{H}$ can be written as

$$
x=\sum_{n \in I} c_{n} x_{n}
$$

where the coefficients $c_{n}$ are computed explicitly by $c_{n}=\left\langle S^{-1} x, x_{n}\right\rangle$. Here $S^{-1}$ is the inverse of the linear operator $S x=\sum_{n \in I}\left\langle x, x_{n}\right\rangle x_{n}$. Observe that the elements of the frame are not necessarily orthogonal, therefore frames can be thought of as over-complete bases.

The possible over-completeness of frames makes them more flexible than orthonormal bases, and therefore a powerful tool in several branches of mathematics [8], [9], [11], [12], [18], [13], [15], [21] and physics [2], [3], [4], [12], [13], [20]. For this reason it is natural to consider frame theory in Hilbert spaces with W-metrics and Krein spaces [1], [14], [17], [16], [20].

A Hilbert space with $W$-metric (or simply $W$-space) $\mathcal{H}_{\mathcal{W}}$ is the completion of $(\mathcal{H},\langle\mathcal{W} \cdot, \cdot\rangle)$, where $\mathcal{W}^{*}=\mathcal{W} \in \mathcal{B}(\mathcal{H})$, and $\operatorname{ker} \mathcal{W}=\{0\}$ [5], [6]. In [14] frames in Hilbert spaces with Wmetrics were introduced and it was showed that the behavior of the frames depends on the boundedness of the linear operator $\mathcal{W}$, and whether or not $0 \in \operatorname{spec}(\mathcal{W})$. Independently of this, the Decomposition Theorem for frames in Hilbert spaces with W-metrics still holds. Similar ideas have been developed independently in [17] and [20].

In [9], [19], [18] there are methods to construct frames in a Hilbert space. Our approach follows the results obtained in [9], [19], [18], [14] in order to construct frames in Hilbert spaces with W-metrics. We present some characterization of frames unitarily equivalent in terms of their respective analysis operators. Furthermore we show that if $\left\{x_{n}\right\}_{n \in \mathbb{N}}$ and $\left\{y_{n}\right\}_{n \in \mathbb{N}}$ are frames for the $\mathrm{W}$-spaces $\mathcal{K}_{G}$ and $\mathcal{H}_{\mathcal{W}}$, respectively, then the coupling of these families is a frame. That is, $\left\{x_{n}\right\}_{n \in \mathbb{N}} \cup\left\{y_{n}\right\}_{n \in \mathbb{N}}$ is a frame for the Hilbert space with W-metric $\mathfrak{K}_{W}=\mathcal{K}_{G} \oplus \mathcal{H}_{\mathcal{W}}$.

We have organized this paper as follows: In Section 2 basic aspects of the Hilbert spaces with W-metrics and frame theory in such spaces are given. In Section 3 we prove the main results of this paper, introduce similar and unitarily equivalent frames and show that two frames are similar if and only if their respective analysis operators have the same range. In addition, we construct frames from a positive and J-adjoint operator (Subsection 3.4). Finally we show that the coupling of two frames is a frame (Subsection $3.3)$. 


\section{Preliminaries}

Let $\mathcal{K}$ be a vector space over $\mathbb{C}$. Consider a sesquilinear form $[\cdot, \cdot]: \mathcal{K} \times \mathcal{K} \longrightarrow \mathbb{C}$. The vector space $(\mathcal{K},[\cdot, \cdot])$ is a Krein space if $\mathcal{K}=\mathcal{K}^{+} \oplus \mathcal{K}^{-}$, where $\left(\mathcal{K}^{+},[\cdot, \cdot]\right),\left(\mathcal{K}^{-},-[\cdot, \cdot]\right)$ are Hilbert spaces, and $\mathcal{K}^{+}, \mathcal{K}^{-}$are orthogonal with respect to $[\cdot, \cdot]$.

On $\mathcal{K}$, define the following inner product:

$$
\left(x_{1}, x_{2}\right)=\left[x_{1}^{+}, x_{2}^{+}\right]-\left[x_{1}^{-}, x_{2}^{-}\right], x_{i}^{ \pm} \in \mathcal{K}^{ \pm}, x_{i}=x_{i}^{+}+x_{i}^{-} .
$$

This inner product turns $(\mathcal{K},(\cdot, \cdot))$ into a Hilbert space, which is called the Hilbert space associated to $\mathcal{K}$. Moreover, there exist unique orthogonal projections onto $\mathcal{K}^{+}$and $\mathcal{K}^{-}$, which are denoted by $P^{+}$and $P^{-}$respectively. The linear bounded operator $J=P^{+}-P^{-}$ is called Fundamental Symmetry. It satisfies

$$
[x, y]_{J}:=[J x, y]=(x, y), \text { and we denote }\|x\|_{J}:=\sqrt{[x, x]_{J}}, \quad x, y \in \mathcal{K} .
$$

The Hilbert space $\left(\mathcal{K},[\cdot, \cdot]_{J}\right)$ is used to study linear operators acting on Krein space $(\mathcal{K},[\cdot, \cdot])$. Topological concepts such as continuity, closedness of operators, spectral theory and so on, refer to the topology induced by the $J$-norm given in (1). Therefore, we may apply the same definitions as in the operator theory of Hilbert spaces. For instance, the adjoint of an operator $T$ in Krein spaces $\left(T^{[*]}\right)$ satisfies $[T(x), y]=\left[x, T^{[*]}(y)\right]$, but we must take into account that $T$ also has an adjoint operator in the Hilbert space $\left(\mathcal{K},[\cdot, \cdot]_{J}\right)$, denoted by $T^{* J}$, where $J$ is the fundamental symmetry in $\mathcal{K}$. The relation between $T^{* J}$ and $T^{[*]}$ is $T^{[*]}=J T^{* J} J$. Moreover, let $\mathcal{K}$ and $\mathcal{K}^{\prime}$ be Krein spaces with fundamental symmetries $J_{\mathcal{K}}$ and $J_{\mathcal{K}^{\prime}}$, respectively. If $T \in \mathcal{B}\left(\mathcal{K}, \mathcal{K}^{\prime}\right)$, then $T^{[*]}=J_{\mathcal{K}} T^{*} J_{\mathcal{K}} J_{\mathcal{K}^{\prime}}$. An operator $T \in \mathcal{B}(\mathcal{K})$ is said to be self-adjoint if $T=T^{[*]}$, and $J$-self-adjoint if $T=T^{* J}$. Furthermore, a linear operator $T$ is said to be invertible if its range and domain are the whole space.

Definition 2.1. Let $V$ be a closed subspace of $\mathcal{K}$. The subspace

$$
V^{[\perp]}=\{x \in \mathcal{K}:[x, y]=0 \text { for all } y \in V\}
$$

is called the $J$-orthogonal complement of $V$ with respect $[\cdot, \cdot]$ (or simply $J$-orthogonal complement of $V$ ).

Definition 2.2. A closed subspace $V$ of $\mathcal{K}$ such that $V \cap V^{[\perp]}=\{0\}$ and $V+V^{[\perp]}=\mathcal{K}$, where $V^{[\perp]}$ is given in (2), is called projectively complete.

Proposition $2.3([6$, Theorem 7.16]). Let $(\mathcal{K},[\cdot, \cdot])$ be a Krein space and let $V$ be a closed subspace of $\mathcal{K}$. The following statements are equivalent:

i) $V$ is projectively complete.

ii) $V$ is a Krein space.

iii) Any vector $y \in \mathcal{K}$ has at least one $J$-orthogonal projection onto $V$.

For more details on Krein spaces, see [6],[7].

Vol. 34, No. 1, 2016] 


\subsection{Hilbert spaces with $W$-metrics}

Definition 2.4. Let $\mathcal{H}$ be a separable Hilbert space with scalar product $\langle\cdot, \cdot\rangle$ and induced norm $\|\cdot\|=\sqrt{\langle\cdot, \cdot\rangle}$. Consider an operator $\mathcal{W}=\mathcal{W}^{*} \in \mathcal{B}(\mathcal{H})$ with $\operatorname{ker} \mathcal{W}=\{0\}$. The sesquilinear form $\mathcal{H}_{\mathcal{W}}$

$$
[\cdot, \cdot]=\langle\mathcal{W}(\cdot), \cdot\rangle
$$

defined on $\mathcal{H}$ is called $\mathrm{W}$-metric, or $W$-inner product, and the operator $\mathcal{W}$ is called Gram operator.

Remark 2.5 (Consequences induced from the Gram operator). Let $\mathcal{W}$ be a Gram operator on $\mathcal{H}$.

i). If $0 \notin \operatorname{spec}(\mathcal{W})$, then

$$
\left\|\mathcal{W}^{-1}\right\|^{-1}\|x\|^{2} \leq\|x\|_{J}^{2} \leq\|\mathcal{W}\|\|x\|^{2}, \quad \forall x \in \mathcal{H} .
$$

Therefore,

$$
\mathcal{H}_{\mathcal{W}}:=\overline{\left(\mathcal{H},[\cdot, \cdot]_{J}\right)} \|^{\|\cdot\|_{J}}=\left(\mathcal{H},[\cdot, \cdot]_{J}\right) .
$$

ii). If $0 \in \operatorname{spec}(\mathcal{W})$, then

$$
\|x\|_{J} \leq \sqrt{\|\mathcal{W}\|}\|x\|, \quad \forall x \in \mathcal{H}
$$

In this case,

$$
\mathcal{H}_{\mathcal{W}}:=\overline{\mathcal{H}}^{\|\cdot\|_{J}} \neq \mathcal{H}
$$

Definition 2.6. Let $(\mathcal{H},\langle\cdot, \cdot\rangle)$ be a separable Hilbert space. The Krein space $\mathcal{H}_{\mathcal{W}}$ is said to be regular if the Gram operator $\mathcal{W}$ is such that $0 \in \rho(\mathcal{W})$. Otherwise, it is said to be singular.

In what follows all the Hilbert spaces are separable. For more details about the regular and singular Krein spaces see [5].

Remark 2.7. Consider the polar decomposition of $\mathcal{W}$,

$$
\mathcal{W}=J|\mathcal{W}|,
$$

where the linear operator $J:(\operatorname{ker}|\mathcal{W}|)^{\perp}=\overline{\operatorname{Rang}|\mathcal{W}|}=\mathcal{H} \rightarrow \overline{\operatorname{Rang} \mathcal{W}}=\mathcal{H}$ is a partial isometry. However, $\operatorname{ker} J=\{0\}$, thus $J=J^{*}$ is a unitary operator.

Definition 2.8. The space $\mathcal{H}_{\mathcal{W}}$ is the Krein space $\left(\overline{\mathcal{H}}^{\|\cdot\|_{J}},[\cdot, \cdot]\right)$ with the sesquilinear form

$$
[x, y]:=\langle\mathcal{W} x, y\rangle
$$

and the $J$-norm $\|\cdot\|_{J}$ given by the scalar product

$$
[x, y]_{J}=[J x, y]=\langle\mathcal{W} J x, y\rangle=\langle|\mathcal{W}| x, y\rangle \quad \forall x, y \in \mathcal{H}_{\mathcal{W}},
$$

where $J$ is the symmetry of Hilbert space $\mathcal{H}$ defined by $\mathcal{W}=J|\mathcal{W}|$. 
Proposition 2.9. If $\mathcal{H}_{\mathcal{W}_{i}}$ is a family of Hilbert spaces with W-metrics indexed by $i \in I$, then the direct sum of the $\mathcal{H}_{\mathcal{W}_{i}}$, denoted

$$
\mathfrak{K}_{\widetilde{W}}=\bigoplus_{i \in I} \mathcal{H}_{\mathcal{W}_{i}},
$$

is a Hilbert space with W-metric, where the Gram operator $\widetilde{W}$ is given by

$$
\widetilde{W}=\bigoplus_{i \in I} \mathcal{W}_{i}
$$

Proof. It is well known if $\mathcal{H}_{i}$ is a family of Hilbert spaces indexed by $i \in I$, then the direct sum

$$
\mathfrak{K}=\bigoplus_{i \in I} \mathcal{H}_{i}
$$

is a Hilbert space with scalar product given by $\langle\cdot, \cdot\rangle_{\mathfrak{K}}=\sum_{i \in I}\langle\cdot, \cdot\rangle_{\mathcal{H}_{i}}$. Thus, if $\widetilde{W}=\bigoplus_{i \in I} \mathcal{W}_{i}$, then the W-metric

$$
\langle\widetilde{W} x, y\rangle_{\mathfrak{K}}=\sum_{i \in I}\left\langle\mathcal{W}_{i} x_{i}, y_{i}\right\rangle_{\mathcal{H}_{i}}, \quad x, y \in \mathfrak{K}
$$

is well defined and bounded. Therefore, the completion $\mathfrak{K}_{\widetilde{W}}$ of $(\mathfrak{K},\langle\widetilde{W} \cdot, \cdot\rangle)$ is a Hilbert space with W-metric and $\mathfrak{K}_{\widetilde{W}}=\bigoplus_{i \in I} \mathcal{H}_{\mathcal{W}_{i}}$.

\section{2. $\ell_{2}(\mathbb{N})$ as a Hilbert space with $W$-metric}

On $\ell_{2}(\mathbb{N})$ consider the following sesquilinear form $[\cdot, \cdot]_{\ell_{2}(\mathbb{N})}$ :

$$
[z, w]_{\ell_{2}(\mathbb{N})}:=\sum_{n=1}^{\infty} \alpha_{n} z_{n} \overline{w_{n}}, \quad z, w \in \ell_{2}(\mathbb{N}),
$$

where $\left(\alpha_{n}\right)_{n \in \mathbb{N}}$ is a sequence of non zero real numbers belonging to $c_{0}(\mathbb{N})$. Let $\left\{\delta_{n}\right\}_{n \in \mathbb{N}}$ be such that $\delta_{n, m}=1$ if $m=n$, and $\delta_{n, m}=0$ for $m \neq n$. It is well known that $\left\{\delta_{n}\right\}_{n \in \mathbb{N}}$ is a orthonormal basis of $\ell_{2}(\mathbb{N})$, and that the diagonal operator $\mathfrak{G}: \ell_{2}(\mathbb{N}) \rightarrow \ell_{2}(\mathbb{N})$ given by

$$
\mathfrak{G} \delta_{n}=\alpha_{n} \delta_{n}, \quad n \in \mathbb{N}
$$

is bounded, self-adjoint and has the spectrum

$$
\operatorname{spec}(\mathfrak{G})=\overline{\left\{\alpha_{n}: n \in \mathbb{N}\right\}} .
$$

Thus, the sesquilinear form $[\cdot, \cdot]_{\ell_{2}(\mathbb{N})}$ is a W-metric on $\ell_{2}(\mathbb{N})$ and satisfies

$$
[z, w]_{\ell_{2}(\mathbb{N})}=\langle\mathfrak{G} z, w\rangle, \quad z, w \in \ell_{2}(\mathbb{N}) .
$$

Since $\mathfrak{G}=\widetilde{J}|\mathfrak{G}|$, where $\widetilde{J} \delta_{n}=\operatorname{sign}\left(\alpha_{n}\right) \delta_{n}$ for each $n \in \mathbb{N}$, we have by Definition 2.8 that

$$
[z, w]_{\tilde{J}}=\langle|\mathfrak{G}| z, w\rangle, \quad z, w \in \ell_{2}(\mathbb{N})
$$


defines a scalar product on $\ell_{2}(\mathbb{N})$. Hence, $\mathfrak{k}_{2, W}(\mathbb{N}):=\overline{\left(\ell_{2}(\mathbb{N}),\langle\mathfrak{G} \cdot, \cdot\rangle\right)}\|\|_{\tilde{J}}$ is a Hilbert space with $\mathrm{W}$-metric with Gram operator $\mathfrak{G}$. Up to unitary equivalence, we may write

$$
\mathfrak{k}_{2, W}(\mathbb{N})=\left\{x \in \mathbb{C}^{\mathbb{N}}: \sum_{n=1}^{+\infty}\left|\alpha_{n}\right|\left|x_{n}\right|^{2} \leq+\infty\right\} .
$$

Remark 2.10. Since $\alpha_{n} \stackrel{n \rightarrow \infty}{\longrightarrow} 0$, one gets $0 \in \operatorname{spec}(\mathfrak{G})$ and $\ell_{2}(\mathbb{N}) \subsetneq \mathfrak{k}_{2, W}(\mathbb{N})$ by Theorem 4.2 in [14].

Proposition 2.11. Let $\mathfrak{k}_{2, W}(\mathbb{N})$ be the Hilbert space with $W$-metric with Gram operator $\mathfrak{G}$ given in (14). Then $U:=\sqrt{|\mathfrak{G}|}$ is a unitary operator $U$ from the Hilbert space $\left(\mathfrak{k}_{2, W}(\mathbb{N}),[\cdot, \cdot]_{J}\right)$ onto $\left(\ell_{2}(\mathbb{N}),\langle\cdot, \cdot\rangle\right)$.

Proof. Let $U=\sqrt{|\mathfrak{G}|}: \mathfrak{k}_{2, W}(\mathbb{N}) \rightarrow \ell_{2}(\mathbb{N})$, where the operator $\mathfrak{G}$ is given in (13). Hence $U$ is an isometry, since

$$
\|U x\|_{\ell_{2}(\mathbb{N})}^{2}=\langle\sqrt{|\mathfrak{G}|} x, \sqrt{|\mathfrak{G}|} x\rangle=\langle|\mathfrak{G}| x, x\rangle=[x, x]_{\tilde{J}}, \quad x \in \mathfrak{k}_{2, W}(\mathbb{N}) .
$$

Moreover, the inverse operator $U^{-1}=(\sqrt{|\mathfrak{G}|})^{-1}: \ell_{2}(\mathbb{N}) \rightarrow \mathfrak{k}_{2, W}(\mathbb{N})$ is well defined. In fact, since $\left\{\alpha_{n}\right\}_{n \in \mathbb{N}}$ is not identically zero and belongs to $c_{0}(\mathbb{N})$, one gets

$$
\left\|U^{-1} y\right\|_{\tilde{J}}^{2}=\lim _{N \rightarrow \infty} \sum_{n=1}^{N}\left|\alpha_{n}\left\|\left.\left(\sqrt{\left|\alpha_{n}\right|}\right)^{-1} y_{n}\right|^{2}=\sum_{n=1}^{+\infty}\left|y_{n}\right|^{2}=\right\| y \|_{\ell_{2}(\mathbb{N})}^{2} .\right.
$$

\subsection{Frames in Hilbert spaces with $W$-metrics}

The next results are based on the frame theory in Krein spaces studied in [14].

Definition 2.12. Let $\mathcal{H}_{\mathcal{W}}$ be a Hilbert space with W-metric. A countable sequence $\left\{x_{n}\right\}_{n \in \mathfrak{N}} \subset \mathcal{H}_{\mathcal{W}}$ is called a frame for $\mathcal{H}_{\mathcal{W}}$, if there exist constants $0<A \leq B<\infty$ such that

$$
A\|x\|_{J}^{2} \leq \sum_{n \in \mathfrak{N}}\left|\left\langle\mathcal{W} x, x_{n}\right\rangle\right|^{2} \leq B\|x\|_{J}^{2} \quad \text { for all } x \in \mathcal{H}_{\mathcal{W}} .
$$

Since we are mostly interested in infinite-dimensional spaces, and since one can always fill up a finite frame with zero elements, we assume that $\mathfrak{N}=\mathbb{N}$ whenever the finiteness of $\mathfrak{N}$ is of no importance

Proposition 2.13 ([14]). Let $\mathcal{H}_{\mathcal{W}}$ be a Hilbert space with $W$-metric. The family $\left\{x_{n}\right\}_{n \in \mathfrak{N}}$ is a frame for $\mathcal{H}_{\mathcal{W}}$, if and only if $\left\{x_{n}\right\}_{n \in \mathfrak{N}}$ is a frame for the associated Hilbert space $\left(\mathcal{H}_{\mathcal{W}},[\cdot, \cdot]_{J}\right)$.

Definition 2.14. Let $\mathcal{H}_{\mathcal{W}}$ be a Hilbert space with $\mathrm{W}$-metric and $\mathfrak{k}_{2, W}(\mathbb{N})$ be the Hilbert space with $\mathrm{W}$-metric $\langle\mathfrak{G} \cdot, \cdot\rangle$ given in (14). If $\left\{x_{n}\right\}_{n \in \mathfrak{N}}$ is a frame for $\mathcal{H}_{\mathcal{W}}$, the linear map

$$
T: \mathfrak{k}_{2, W}(\mathbb{N}) \rightarrow \mathcal{H}_{\mathcal{W}}, \quad T\left(y_{n}\right)_{n \in \mathfrak{N}}=\sum_{n \in \mathfrak{N}} \sqrt{\left|\alpha_{n}\right|} y_{n} x_{n}
$$

is called pre-frame operator. 
Remark 2.15. Let $\left\{x_{n}\right\}_{n \in \mathfrak{N}}$ be a frame for a Hilbert space $\mathcal{H}_{W(\Lambda)}$ with a W-metric. Then, for every $\left(y_{n}\right)_{n \in \mathfrak{N}} \in \mathfrak{k}_{2, W}(\mathbb{N})$ we have

$$
\begin{aligned}
\left\|T\left(y_{n}\right)_{n \in \mathfrak{N}}\right\|_{J} & =\sup _{\|x\|_{J}=1}\left|\left[T\left(y_{n}\right)_{n \in \mathfrak{N}}, x\right]\right| \leq \sup _{\|x\|_{J}=1} \sum_{n \in \mathfrak{N}} \sqrt{\left|\alpha_{n}\right|}\left|y_{n}\right|\left|\left[x, x_{n}\right]\right| \\
& \leq \sup _{\|x\|_{J}=1}\left(\sum_{n \in \mathfrak{N}}\left|\alpha_{n}\right|\left|y_{n}\right|^{2}\right)^{1 / 2}\left(\sum_{n \in \mathfrak{N}}\left|\left[x, x_{n}\right]\right|^{2}\right)^{1 / 2} \\
& \leq\left\|\left(y_{n}\right)_{n \in \mathfrak{N}}\right\|_{\widetilde{J}} \sqrt{B} .
\end{aligned}
$$

Thus, $\|T\| \leq \sqrt{B}$.

The following result provides a criterion for a family $\left\{x_{n}\right\}_{n \in \mathfrak{N}}$ to be a frame for $\mathcal{H}_{\mathcal{W}}$ (see [14]) and is an analogue to the Hilbert case.

Proposition 2.16 ([14]). Let $\mathcal{H}_{\mathcal{W}}$ be a Hilbert space with $W$-metric. The family $\left\{x_{n}\right\}_{n \in \mathfrak{N}}$ is a frame for $\mathcal{H}_{\mathcal{W}}$, if and only if $T$ is well defined (i.e., bounded) and surjective.

Remark 2.17. It is easily seen that for all $\left(y_{n}\right)_{n \in \mathfrak{N}} \in \mathfrak{k}_{2, W}(\mathbb{N})$

$$
\begin{aligned}
{\left[T\left(y_{n}\right)_{n \in \mathfrak{N}}, k\right] } & =\sum_{n \in \mathfrak{N}} \sqrt{\left|\alpha_{n}\right|} y_{n}\left[x_{n}, k\right]=\left\langle U\left(y_{n}\right)_{n \in \mathfrak{N}},\left(\left[k, x_{n}\right]\right)_{n \in \mathfrak{N}}\right\rangle_{\ell_{2}(\mathbb{N})} \\
& =\left\langle\left(y_{n}\right)_{n \in \mathfrak{N}}, U^{-1}\left(\left[k, x_{n}\right]\right)\right\rangle_{\mathfrak{k}_{2, W}(\mathbb{N})}=\left[\left(y_{n}\right)_{n \in \mathfrak{N}}, \widetilde{J} U^{-1}\left(\left[k, x_{n}\right]\right)\right], k \in \mathcal{K} .
\end{aligned}
$$

Thus, the adjoint operator $T^{[*]}$ of $T$ is given by

$$
T^{[*]} x=\tilde{J} U^{-1}\left(\left[x, x_{n}\right]\right)_{n \in \mathfrak{N}}, \quad x \in \mathcal{H}_{\mathcal{W}},
$$

where $U^{-1}=\sqrt{|\mathfrak{G}|}^{-1}$. The operator $T^{[*]}$ is called analysis operator.

Definition 2.18 ([14]). Let $\mathcal{H}_{\mathcal{W}}$ be a Hilbert space with W-metric, $\left(\mathfrak{k}_{2, W}(\mathbb{N}),\langle\mathfrak{G} \cdot, \cdot\rangle\right)$ be a Hilbert space with W-metric given in (14), and $\left\{k_{n}\right\}_{n \in \mathfrak{N}} \subset \mathcal{H}_{\mathcal{W}}$ a frame for $\mathcal{H}_{\mathcal{W}}$. The operator

$$
S:=T \tilde{J} T^{[*]}
$$

is called frame operator.

Let $\left\{x_{n}\right\}_{n \in \mathfrak{N}}$ be a frame for $\mathcal{H}_{\mathcal{W}}$. The linear operator $T_{0}: \ell_{2}(\mathbb{N}) \rightarrow \mathcal{H}_{\mathcal{W}}$ given by the rule

$$
T_{0}\left(y_{n}\right)=\sum_{n \in \mathfrak{N}} y_{n} x_{n}
$$

is well defined, and its adjoint operator is $T_{0}^{[*]} k=\left(\left[k, x_{n}\right]\right)_{n \in \mathfrak{N}}$. Observe that the preframe operator $T$ given in (18) is such that

$$
T=T_{0} U, \quad T^{[*]}=\widetilde{J} U^{-1} T_{0}^{[*]}, \quad \text { where } \quad U=\sqrt{|\mathfrak{G}|} .
$$

It follows immediately from $(21),(22)$, and $\tilde{J}^{2}=U U^{-1}=\mathrm{id}_{\ell_{2}(\mathbb{N})}$ that

$$
S x=\sum_{n \in \mathfrak{N}}\left[x_{n}, x\right] x_{n}, \quad x \in \mathcal{H}_{\mathcal{W}} .
$$


Furthermore, $S$ is self-adjoint and invertible. If we put $\alpha_{n}=1$ for each $n \in \mathbb{N}$ in (12), then $\left(\mathfrak{k}_{2, W}(\mathbb{N}),[\cdot, \cdot]\right)=\left(\ell_{2}(\mathbb{N}),\langle\cdot, \cdot\rangle\right)$, and the frame operator is $S=T T^{*}$, exactly as in the Hilbert space case.

Proposition 2.19. Let $\mathcal{H}_{\mathcal{W}}$ be a Hilbert space with $W$-metric. If $\left\{x_{n}\right\}_{n \in \mathfrak{N}}$ is a frame for $\mathcal{H}_{\mathcal{W}}$, then the image of the analysis operator, denoted by $\operatorname{Rang} T^{[*]}$, is a Krein subspace of $\mathfrak{k}_{2, W}(\mathbb{N})$.

Proof. Since $\left\{x_{n}\right\}_{n \in \mathfrak{N}}$ is a frame for $\mathcal{H}_{W(\Lambda)}$, there is $A>0$ such that

$$
\begin{aligned}
A\|k\|_{J}^{2} & \leq \sum_{n \in \mathfrak{N}}\left|\left[k, x_{n}\right]\right|^{2}=[S k, k]=\left[T \widetilde{J} T^{[*]} k, k\right]=\left[\widetilde{J} T^{[*]} k, T^{[*]} k\right]_{\mathfrak{k}_{2, W}(\mathbb{N})} \\
& \leq\left[T^{[*]} k, T^{[*]} k\right]_{\widetilde{J}}=\left\|T^{[*]} k\right\|_{\tilde{J}}^{2}, \quad k \in \mathcal{H}_{W} .
\end{aligned}
$$

Thus, $A\|k\|_{J} \leq\left\|T^{[*]} k\right\|_{\tilde{J}}$ for each $k \in \mathcal{H}_{W}$; therefore, $\operatorname{Rang} T^{[*]}$ is a closed subspace of $\mathfrak{k}_{2, W}(\mathbb{N})$. On the other hand, if $x \in \operatorname{Rang} T^{[*]} \cap\left(\operatorname{Rang} T^{[*]}\right)^{[\perp]}$, then $[x, x]_{\mathfrak{k}_{2, W}(\mathbb{N})}=0$. Since $x=T^{[*]} y$ for some $y \in \mathcal{H}_{\mathcal{W}}$, we have that $y \in \operatorname{ker} T^{[*]}$, and by injectivity of $T^{[*]}$ (Proposition 2.16), one gets that $x=0$. Hence, Rang $T^{[*]}$ is a Krein subspace of $\mathfrak{k}_{2, W}(\mathbb{N})$.

\section{Main results}

\subsection{Similar frames in Hilbert spaces with $W$-metrics}

Definition 3.1. Let $\left(\mathcal{H}_{\mathcal{W}},[\cdot, \cdot]_{\mathcal{H}}\right)$ and $\left(\mathcal{K}_{G},[\cdot, \cdot]_{\mathcal{K}}\right)$ be Hilbert spaces with $W$-metrics and Gram operators $\mathcal{W}$ and $G$, respectively. Two frames $\left\{x_{n}\right\}_{n \in \mathfrak{N}}$ and $\left\{y_{n}\right\}_{n \in \mathfrak{N}}$ for $\mathcal{H}_{W}$ and $\mathcal{K}_{G}$, respectively, are said to be similar if there exists an invertible operator $\mathcal{U}: \mathcal{H}_{W} \rightarrow$ $\mathcal{K}_{G}$ such that $\mathcal{U} y_{n}=x_{n}$ for $n \in \mathfrak{N}$. The frames are called unitarily equivalent if $U$ is a unitary operator from $\mathcal{H}_{\mathcal{W}}$ onto $\mathcal{K}_{G}$.

In [14] it was proven that the behavior of frames in Hilbert spaces with W-metrics depends on whether the Gram operator is bounded or not. However, for any unitary operator $U: \mathcal{H} \rightarrow \mathcal{H}_{\mathcal{W}},\left\{x_{n}\right\}_{n \in \mathfrak{N}}$ is a frame for $\mathcal{H}$ if and only if $\left\{U x_{n}\right\}_{n \in \mathfrak{N}}$ is a frame for $\mathcal{H}_{\mathcal{W}}$.

Remark 3.2. Note that $\mathcal{H}$ and $\mathcal{H}_{\mathcal{W}}$ are unitarily equivalent, since both are separable.

The following result provides a criterion for a couple of frames to be similar. It is an analogue to Deguang and Larson's result [19] for Hilbert spaces.

Theorem 3.3. Let $\left\{x_{n}\right\}_{n \in \mathfrak{N}}$, and $\left\{y_{n}\right\}_{n \in \mathfrak{N}}$ be frames for Hilbert spaces with $W$-metrics $\left(\mathcal{H}_{\mathcal{W}},[\cdot, \cdot]_{\mathcal{H}}\right)$ and $\left(\mathcal{K}_{G},[\cdot, \cdot]_{\mathcal{K}}\right)$, respectively. Let $T_{\mathcal{H}_{\mathcal{W}}}^{[*]_{\mathcal{H}}}$ and $T_{\mathcal{K}_{G}}^{[*]_{\mathcal{K}_{G}}}$ be the analysis operators for $\left\{x_{n}\right\}_{n \in \mathfrak{N}}$ and $\left\{y_{n}\right\}_{n \in \mathfrak{N}}$, respectively. The frames $\left\{x_{n}\right\}_{n \in \mathfrak{N}}$ and $\left\{y_{n}\right\}_{n \in \mathfrak{N}}$ are similar if and only if $T_{\mathcal{H}_{\mathcal{W}}}^{\left[* \mathcal{H}_{\mathcal{W}}\right.}$ and $T_{\mathcal{K}_{G}}^{[*]_{\mathcal{K}_{G}}}$ have the same range.

Proof. Let $T_{\mathcal{H}}^{\left[* \mathcal{H}_{\mathcal{W}}\right.}$ and $T_{\mathcal{K}_{G}}^{[*]_{\mathcal{K}}}$ be the analysis operator for $\left\{x_{n}\right\}_{n \in \mathfrak{N}}$ and $\left\{y_{n}\right\}_{n \in \mathfrak{N}}$, respectively. Suppose that $\left\{x_{n}\right\}_{n \in \mathfrak{N}}$ and $\left\{y_{n}\right\}_{n \in \mathfrak{N}}$ are similar; then, there is an invertible operator $\mathcal{U}: \mathcal{H}_{\mathcal{W}} \rightarrow \mathcal{K}_{G}$ such that $\mathcal{U} x_{n}=y_{n}$. Hence,

$$
T_{\mathcal{K}_{G}}^{\left[* \mathcal{K}_{G}\right.} y=\widetilde{J} U^{-1}\left(\left[y, \mathcal{U} x_{n}\right]\right)_{n \in \mathfrak{N}}=\widetilde{J} U^{-1}\left(\left[\mathcal{U}^{[*]} y, x_{n}\right]\right)_{n \in \mathfrak{N}}=T_{\mathcal{H}_{\mathcal{W}}}^{[*]_{\mathcal{W}}} \mathcal{U}^{[*]} y .
$$


Now, since $\mathcal{U}^{[*]}$ is invertible, we concluded that $T_{\mathcal{K}_{G}}^{\left[* \mathcal{K}_{G}\right.}\left(\mathcal{K}_{G}\right)=T_{\mathcal{H}_{\mathcal{W}}}^{[*]_{\mathcal{H}}} \mathcal{U}^{[*]}\left(\mathcal{K}_{G}\right)$. Thus, $\operatorname{Rang} T_{\mathcal{K}_{G}}^{[*]_{\mathcal{K}_{G}}}=\operatorname{Rang} T_{\mathcal{H}_{\mathcal{W}}}^{[*]_{\mathcal{H}}}$.

Conversely, suppose that $\operatorname{Rang} T_{\mathcal{K}_{G}}^{[*]_{\mathcal{K}_{G}}}=\operatorname{Rang} T_{\mathcal{H}_{\mathcal{W}}}^{[*]_{\mathcal{H}}}=V$. Let $U$ be the unitary operator given in (16). Note that $\left.T_{\mathcal{H}}\right|_{U \widetilde{J} V}$ y $\left.T_{\mathcal{K}_{G}}\right|_{U \widetilde{J} V}$ are invertible. Thus, the operator

$$
\mathcal{U}=T_{\mathcal{K}_{G}}\left(U \widetilde{J} T_{\mathcal{H}_{\mathcal{W}}}^{[*]]_{\mathcal{W}}} T_{\mathcal{H}_{\mathcal{W}}}\right)^{-1} U \widetilde{J} T_{\mathcal{H}_{\mathcal{W}}}^{[*]_{\mathcal{H}}}: \mathcal{H}_{\mathcal{W}} \rightarrow \mathcal{K}_{G}
$$

is well defined and invertible. By Proposition 2.19 the closed subspace $V$ is projectively complete, so there exists a unique $J$-orthogonal projection $P$ from $\mathfrak{k}_{2, W}(\mathbb{N})$ on $V$. If $y \in V^{[\perp]_{2, W}(\mathbb{N})}$, then for every $z \in \mathcal{K}_{G}$ and $T_{\mathcal{K}_{G}}^{\left[{ }^{*} \mathcal{K}_{G}\right.} z \in V$, we get

$$
0=[x, y]_{\mathfrak{k}_{2, W}(\mathbb{N})}=\left[T_{\mathcal{K}_{G}}^{[*]_{\mathcal{K}_{G}}} z, y\right]_{\mathfrak{k}_{2, W}(\mathbb{N})}=\left[z, T_{\mathcal{K}_{G}} y\right]_{\mathcal{K}_{G}} .
$$

Since $y$ was arbitrary, we conclude that $T_{\mathcal{K}_{G}}\left(V^{[\perp]_{\mathfrak{e}_{2, W}}(\mathbb{N})}\right)=\{0\}$. Analogously, we have $T_{\mathcal{H}_{\mathcal{W}}}\left(V^{[\perp]_{\mathfrak{e}_{2, W}(\mathbb{N})}}\right)=\{0\}$. Now, consider $\left\{v_{m}\right\}_{m \in \mathfrak{N}}$, where $v_{m, j}=\delta_{m, j}$. By the definition of pre-frame operator (18), we get $T_{\mathcal{H}_{\mathcal{W}}} P v_{m}=T_{\mathcal{H}_{\mathcal{W}}} v_{m}=\sum_{j \in \mathfrak{N}} \delta_{m, j} x_{m}=x_{m}$. On the other hand, it also satisfies that $y_{m}=T_{\mathcal{K}_{G}} v_{m}=T_{\mathcal{K}_{G}} P v_{m}$. Hence,

$$
\begin{aligned}
\mathcal{U} x_{m}=\mathcal{U} T_{\mathcal{H}_{\mathcal{W}}} P v_{m} & =T_{\mathcal{K}_{G}}\left(U \widetilde{J} T_{\mathcal{H}_{\mathcal{W}}}^{[*]_{\mathcal{H}}} T_{\mathcal{H}_{\mathcal{W}}}\right)^{-1} U \widetilde{J} T_{\mathcal{H}_{\mathcal{W}}}^{[*] \mathcal{H}_{\mathcal{W}}} T_{\mathcal{H}_{\mathcal{W}}} P v_{m} \\
& =T_{\mathcal{K}_{G}} P v_{m}=y_{m} .
\end{aligned}
$$

\subsection{Construction of frames}

Proposition 3.4 (Construction of frames with an operator on finite-dimensional Krein spaces). Let $(\mathcal{K},[\cdot, \cdot])$ be a $N$-dimensional Krein space with fundamental symmetry $J$, and $S_{0}$ be a J-self-adjoint positive operator with respect to $[\cdot, \cdot]_{J}$. Let $\lambda_{1} \geq \lambda_{2} \geq \ldots \geq \lambda_{N}>0$ be the eigenvalues of $S_{0}$. Fix $k \geq N$ and real numbers $a_{1} \geq a_{2} \geq \cdots \geq a_{k}>0$. The following statements are equivalent:

(i) For every $1 \leq j \leq N$,

$$
\sum_{n=1}^{j} a_{n}^{2} \leq \sum_{n=1}^{j} \lambda_{n}, \quad \sum_{n=1}^{k} a_{n}^{2} \leq \sum_{n=1}^{N} \lambda_{n} .
$$

(ii) There is a frame $\left\{x_{n}\right\}_{n=1}^{k}$ for the Hilbert space $\left(\mathcal{K},[\cdot, \cdot]_{J}\right)$ with frame operator $S_{0}$ and $\left\|x_{n}\right\|_{J}=a_{n}$, for all $n=1, \ldots, k$.

(iii) There is a frame $\left\{x_{n}\right\}_{n=1}^{k}$ for the Krein space $\mathcal{K}$ with frame operator $S_{0} J$ and $\left\|x_{n}\right\|_{J}=a_{n}$, for all $n=1, \ldots, k$.

(iv) There is a frame $\left\{J x_{n}\right\}_{n=1}^{k}$ for the the Krein space $\mathcal{K}$ with frame operator $J S_{0}$ and $\left\|x_{n}\right\|_{J}=a_{n}$, for all $n=1, \ldots, k$.

Vol. 34, No. 1, 2016] 
(v) There is a frame $\left\{J x_{n}\right\}_{n=1}^{k}$ for the Hilbert space $\left(\mathcal{K},[\cdot, \cdot]_{J}\right)$ with frame operator $J S_{0} J$ and $\left\|x_{n}\right\|_{J}=a_{n}$, for all $n=1, \ldots, k$.

Proof. The equivalence between (i) and (ii) is proved in [9]. By Proposition 2.13 and the remarks following Theorem 3.6 in [14], we have the equivalences (ii) $\leftrightarrow$ (iii) $\leftrightarrow$ (iv) $\leftrightarrow$ (v). Now we only need to find the relationship of the respective frame operators with the operator $S_{0}$.

Since $S_{0}$ is the frame operator corresponding to the family $\left\{x_{n}\right\}_{n=1}^{k}$, we have for every $x \in \mathcal{K}$

$$
\sum_{n=1}^{k}\left[x, x_{n}\right] x_{n}=\sum_{n=1}^{k}\left[J x, x_{n}\right]_{J} x_{n}=S_{0} J x .
$$

Therefore, $S_{1}:=S_{0} J$ is the frame operator for the frame $\left\{x_{n}\right\}_{n=1}^{k}$ in the Krein space $\mathcal{K}$. Next, for every $x \in \mathcal{K}$, one gets

$$
\sum_{n=1}^{k}\left[x, J x_{n}\right] J x_{n}=J\left(\sum_{n=1}^{k}\left[x, x_{n}\right]_{J} x_{n}\right)=J S_{0} x .
$$

Thus, $S_{2}:=J S_{0}$ is the frame operator for the frame $\left\{J x_{n}\right\}_{n=1}^{k}$ in the Krein space $\mathcal{K}$.

Finally, for every $x \in \mathcal{K}$ we have that

$$
\sum_{n=1}^{k}\left[x, J x_{n}\right]_{J} J x_{n}=J\left(\sum_{n=1}^{k}\left[J x, x_{n}\right]_{J} x_{n}\right)=J S_{0} J x .
$$

Hence, $S_{3}:=J S_{0} J$ is the frame operator for the frame $\left\{J x_{n}\right\}_{n=1}^{k}$ in the Hilbert space $\left(\mathcal{K},[\cdot, \cdot]_{J}\right)$.

Consider a Hilbert space $(\mathcal{H},\langle\cdot, \cdot\rangle)$, with a frame $\left\{x_{n}\right\}_{n \in \mathfrak{N}}$ and frame bounds $0<A \leq B$. The operator frame $S: \mathcal{H} \rightarrow \mathcal{H}$ given by

$$
S x=\sum_{n \in \mathfrak{N}}\left\langle x, x_{n}\right\rangle x_{n}, \quad x \in \mathcal{H}
$$

is bounded, invertible and $A \operatorname{Id} \leq S \leq B$ Id (see for example [10]). Therefore, the Hilbert space with $\mathrm{W}$-metric $\mathcal{H}_{\mathcal{W}}=\left(\mathcal{H},\|\cdot\|_{J}\right)$ is a regular Krein space with frame $\left\{x_{n}\right\}_{n \in \mathfrak{N}}$, where the norm $\|\cdot\|_{J}$ is given by $\|\cdot\|_{J}=\sqrt{\langle S \cdot, \cdot\rangle}$ and satisfies (4).

Now we pose the following problem: find a Hilbert space with W-metric $\mathcal{H}_{\mathcal{W}}$ such that $\mathcal{H} \subsetneq \mathcal{H}_{\mathcal{W}}$, and construct a frame $\left\{y_{n}\right\}_{n \in \mathfrak{N}}$ for $\mathcal{H}_{\mathcal{W}}$ unitarily equivalent or similar to $\left\{x_{n}\right\}_{n \in \mathfrak{N}}$. The next lemma solves the problem.

Lemma 3.5. Let $(\mathcal{H},\langle\cdot, \cdot\rangle)$ be a Hilbert space and $\left\{e_{n}\right\}_{n \in \mathfrak{N}}$ be a orthonormal basis for $\mathcal{H}$. If $\mathfrak{G}$ is the linear operator defined in (14), then the linear operator $\mathcal{W}_{\mathfrak{G}}: \mathcal{H} \rightarrow \mathcal{H}$ given by

$$
\mathcal{W}_{\mathfrak{G}}\left(\sum_{n \in \mathfrak{N}} x_{n} e_{n}\right)=\sum_{n \in \mathfrak{N}} \alpha_{n} x_{n} e_{n}
$$

is well defined, bounded, $\mathcal{W}_{\mathfrak{G}}^{*}=\mathcal{W}_{\mathfrak{G}}, \operatorname{ker} \mathcal{W}_{\mathfrak{G}}=\{0\}$ and $0 \in \operatorname{spec}\left(\mathcal{W}_{\mathfrak{G}}\right)$. 
Proof. Let $x=\sum_{n \in \mathfrak{N}} x_{n} e_{n}$. Then $x \in \operatorname{ker}\left(\mathcal{W}_{\mathfrak{G}}\right)$ if and only if $\sum_{n \in \mathfrak{N}} \alpha_{n} x_{n} e_{n}=0$. Therefore $\alpha_{n} x_{n}=0$ for all $n \in \mathbb{N}$, i.e., $x_{n}=0$ for all $n \in \mathbb{N}$. Thus, $x=0$. Moreover $0 \in \operatorname{spec}\left(\mathcal{W}_{\mathfrak{G}}\right), \operatorname{since} \operatorname{spec}\left(\mathcal{W}_{\mathfrak{G}}\right)=\overline{\left\{\alpha_{n}: n \in \mathbb{N}\right\}}$ and $\alpha_{n} \stackrel{n \rightarrow \infty}{\longrightarrow} 0$.

Proposition 3.6. If $(\mathcal{H},\langle\cdot, \cdot\rangle)$ is a Hilbert space and $\left\{x_{n}\right\}_{n \in \mathfrak{N}}$ is a frame for $\mathcal{H}$, then there exist a Hilbert space with $W$-metric $\mathcal{H}_{\mathcal{W}}$ such that $\mathcal{H} \subsetneq \mathcal{H}_{\mathcal{W}}$, and a frame $\left\{y_{n}\right\}_{n \in \mathfrak{N}}$ for $\mathcal{H}_{\mathcal{W}}$ which is unitarily equivalent to $\left\{x_{n}\right\}_{n \in \mathfrak{N}}$.

Proof. Let $(\mathcal{H},\langle\cdot, \cdot\rangle)$ be a Hilbert space and $\left\{x_{n}\right\}_{n \in \mathfrak{N}}$ be a frame for $\mathcal{H}$. If $\left\{e_{n}\right\}_{n \in \mathfrak{N}}$ is a orthonormal basis for $\mathcal{H}$, then by Lemma 3.5 the operator $\mathcal{W}_{\mathfrak{G}}$ given in $(25)$ is well defined, bounded, $\mathcal{W}_{\mathfrak{G}}^{*}=\mathcal{W}_{\mathfrak{G}}$ and $\operatorname{ker} \mathcal{W}_{\mathfrak{G}}=\{0\}$. Thus, we may consider $\mathcal{H}_{\mathcal{W}}$, the Hilbert space with W-metric with Gram operator $\mathcal{W}_{\mathfrak{G}}$. Now $\mathcal{H} \subsetneq \mathcal{H}_{\mathcal{W}}$ and this is an equality if and only if $0 \notin \operatorname{spec}\left(\mathcal{W}_{\mathfrak{G}}\right)$ (see [14, Theorem 4.2]). On the other hand, the existence of a frame unitarily equivalent to $\left\{x_{n}\right\}_{n \in \mathfrak{N}}$ follows from the facts that $\mathcal{H}$ and $\mathcal{H}_{\mathcal{W}}$ are unitarily equivalent (since both are separable), and that the unitary operators transfer frames into frames with the same frame bounds.

\subsection{The coupling of frames}

Proposition 3.7. Let $\mathcal{K}_{G}$ and $\mathcal{H}_{\mathcal{W}}$ be Hilbert spaces with $W$-metrics, and $\left\{x_{n}\right\}_{n \in \mathfrak{N}}$, $\left\{y_{n}\right\}_{n \in \mathfrak{N}}$ be frames for $\mathcal{K}_{G}, \mathcal{H}_{\mathcal{W}}$, respectively. Denote by $S_{\mathcal{K}_{G}}, S_{\mathcal{H}_{\mathcal{W}}}$ their corresponding frame operators. Then $\left\{x_{n}\right\}_{n \in \mathfrak{N}} \cup\left\{y_{n}\right\}_{n \in \mathfrak{N}}$ is a frame for $\mathfrak{K}_{W}:=\mathcal{K}_{G} \oplus \mathcal{H}_{\mathcal{W}}$, and the corresponding frame operator is given by

$$
S_{\mathfrak{K}_{W}}=S_{\mathcal{K}_{G}} \oplus S_{\mathcal{H}_{\mathcal{W}}} .
$$

Proof. Set $\mathfrak{K}_{W}=\mathcal{K}_{G} \oplus \mathcal{H}_{\mathcal{W}}$. By Proposition $2.9, \mathfrak{K}_{W}$ is a Hilbert space with W-metric, where the Gram operator is given by $W=G \oplus \mathcal{W}$. Now, consider the pre-frame operators

$$
\begin{gathered}
T_{\mathcal{K}_{G}}: \mathfrak{k}_{2, W_{1}}(\mathbb{N}) \rightarrow \mathcal{K}_{G}, \\
T_{\mathcal{H}_{\mathcal{W}}}: \mathfrak{k}_{2, W_{2}}(\mathbb{N}) \rightarrow \mathcal{H}_{\mathcal{W}},
\end{gathered}
$$

as defined in (18). Let $\mathcal{V}: \mathfrak{k}_{2, W_{3}}(\mathbb{N}) \rightarrow \mathfrak{k}_{2, W_{1}}(\mathbb{N}) \oplus \mathfrak{k}_{2, W_{2}}(\mathbb{N})$ be any unitary isometry, i.e.,

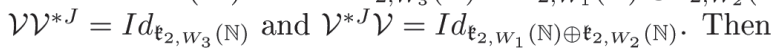

$$
\left(T_{\mathcal{K}_{G}} \oplus T_{\mathcal{H}_{\mathcal{W}}}\right) \mathcal{V}: \mathfrak{k}_{2, W_{3}} \rightarrow \mathcal{K}_{G} \oplus \mathcal{H}_{\mathcal{W}}
$$

is bounded and surjective, hence it is a pre-frame operator for the frame $\left\{x_{n}\right\}_{n \in \mathfrak{N}} \cup$ $\left\{y_{n}\right\}_{n \in \mathfrak{N}}$ for $\mathfrak{K}_{W}=\mathcal{K}_{G} \oplus \mathcal{H}_{\mathcal{W}}$. Then, by Definition 2.18,

$$
\begin{aligned}
& S_{\mathfrak{K}_{W}}=\left(T_{\mathcal{K}_{G}} \oplus T_{\mathcal{H}_{\mathcal{W}}}\right) \mathcal{V}{\widetilde{\mathcal{J}_{2}, W_{3}}}_{\mathfrak{N})} \mathcal{V}^{[*]}\left(T_{\mathcal{K}_{G}} \oplus T_{\mathcal{H}_{\mathcal{W}}}\right)^{[*]}
\end{aligned}
$$

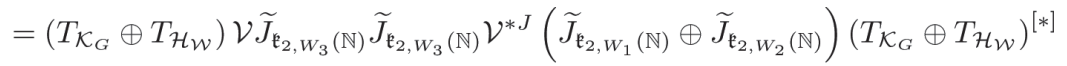

$$
\begin{aligned}
& =\left(T_{\mathcal{K}_{G}} \oplus T_{\mathcal{H}_{\mathcal{W}}}\right)\left(\widetilde{J}_{\mathfrak{k}_{2, W_{1}}(\mathbb{N})} \oplus{\widetilde{\mathfrak{k}_{2}, W_{2}}}_{\mathbb{N})}\right)\left(T_{\mathcal{K}_{G}}^{[*]} \oplus T_{\mathcal{H}_{\mathcal{W}}}^{[*]}\right) \\
& =\left(T_{\mathcal{K}_{G}} \widetilde{J}_{\mathfrak{k}_{2, W_{1}}(\mathbb{N})} T_{\mathcal{K}_{G}}^{[*]}\right) \oplus\left(T_{\mathcal{H}_{\mathcal{W}}} \widetilde{J}_{\mathfrak{k}_{2, W_{2}}(\mathbb{N})} T_{\mathcal{H}_{\mathcal{W}}}^{[*]}\right) \\
& =S_{\mathcal{K}_{G}} \oplus S_{\mathcal{H}_{\mathcal{W}}} \text {. }
\end{aligned}
$$

Vol. 34, No. 1, 2016] 


\section{Acknowledgement}

The second author is grateful to the coauthors, to Ricardo Cedeño (R.I.P.) and to the Universidad Surcolombiana for their hospitality during his stay in the city of Neiva. The authors wish to express his gratitude to the Universidad de Sucre. The work on the paper was partially supported by the project Extensión de Frames en espacios de métrica indefinida. We also thank an anonymous referee for constructive comments, recommendations and useful suggestions.

\section{References}

[1] Acosta-Humánez P., Esmeral K. and Ferrer O., "Frames of subspaces in Hilbert spaces with W-metrics", An. Stiint. Univ. Ovidius Constanta Ser. Mat. 23 (2015), No. 2, 5-22.

[2] Ali S.T., Antoine J.P. and Gazeau J.P., Coherent states, wavelets and their generalizations, Springer-Verlag, New York, 2000.

[3] Ali S.T., Antoine J.P. and Gazeau J.P., "Continuous frames in Hilbert space", Ann. Physics 222 (1993), No. 1, 1-37.

[4] Ali S.T., Antoine J.P. and Gazeau J.P., "Square integrability of group representations on homogeneus spaces. I. Reproducing triples and frames", Ann. Inst. H. Poincaré Phys. Théor. 55 (1991), No. 4, 829-855.

[5] Azizov T.Y. and Iokhvidov I.S., "Linear operators in Hilbert spaces with $G$-metric", Russian Math. Surveys 26 (1971), No. 4 45-97.

[6] Azizov T.Y. and Iokhvidov I.S., Linear operator in spaces with an indefinite metric, John Wiley \& Sons Ltd., Chichester, 1989.

[7] Bognár J., Indefinite inner product spaces, Springer-Verlag, New York-Heidelberg, 1974.

[8] Casazza P.G., "The art of frame theory", Taiwanese J. Math. 4 (2000), No. 2, 129-201.

[9] Casazza P.G. and Leon M.T., "Existence and construction of finite frames with a given frame operator", Int. J. Pure Appl. Math. 63 (2010), No. 2, 149-157.

[10] Christensen O., An introduction to frames and Riesz bases, Applied and Numerical Harmonic Analysis, Birkhäuser Boston Inc., Boston, MA, 2003.

[11] Daubechies I., "The wavelet transform, time-frequency localization and signal analysis", IEEE Trans. Inform. Theory 36 (1990), No. 5, 961-1005.

[12] Daubechies I., Grossmann A. and Meyer Y., "Painless nonorthogonal expansions", J. Math. Phys. 27 (1986), No. 5, 1271-1283.

[13] Duffin R.J. and Schaeffer A.C., "A class of nonharmonic Fourier series", Trans. Amer. Math. Soc. 72 (1952), 341-366.

[14] Esmeral K., Ferrer O. and Wagner E., "Frames in Krein spaces arising from a non-regular W-metric", Banach J. Math. Anal. 9 (2015), No. 1, 1-16.

[15] Găvruţa P., "On the duality of fusion frames", J. Math. Anal. Appl. 333 (2007), No. 2, 871-879. 
[16] Giribet J.I., Maestripieri A., Martínez Pería F. and Massey P.G., "On a family of frames for Krein spaces", arXiv:1112.1632v1.

[17] Giribet J.I., Maestripieri A., Martínez Pería F. and Massey P.G., "On frames for Krein spaces", J. Math. Anal. Appl. 393 (2012), No. 1, 122-137.

[18] Han D., Kornelson K., Larson D.R. and Weber E., Frames for undergraduates, American Mathematical Society, Providence, RI, 2007.

[19] Han D. and Larson D.R., "Frames, bases and group representations", Mem. Amer. Math. Soc. 147 (2000) No. 697, 94 pp.

[20] Peng I. and Waldron S., "Signed frames and Hadamard products of Gram matrices", Linear Algebra Appl. 347 (2002), 131-157.

[21] Rahimi A., Najati, A. and Dehghan Y.N., "Continuous frames in Hilbert spaces", Methods Funct. Anal. Topology 12 (2006), No. 2, 170-182. 